\title{
Arterial spin labeling: Adaptive thresholding for epileptogenic zone detection in MRI-negative epilepsy
}

\section{Martin Gajdoš}

CEITEC - Central European Institute of Technology, Masaryk University

\section{Pavel Říha}

CEITEC - Central European Institute of Technology, Masaryk University

\section{Martin Kojan}

CEITEC - Central European Institute of Technology, Masaryk University

\section{Irena Doležalová}

Brno Epilepsy Center, Department of Neurology, St. Anne's University Hospital and Medical Faculty of Masaryk University

\section{Henk-Jan Mutsaerts}

Amsterdam University Medical Center, VU University Medical Center, Amsterdam Neuroscience

\section{Jan Petr}

Helmholtz-Zentrum Dresden-Rossendorf, Institute of Radiopharmaceutical Cancer Research

Ivan Rektor ( $\nabla$ irektor@med.muni.cz)

Brno Epilepsy Center, Department of Neurology, St. Anne's University Hospital and Medical Faculty of Masaryk University

\section{Research Article}

Keywords: Drug-resistant Epilepsy, Hypoperfusion, Minimal Product Criterion, Minimal Distance Criterion, Elbow Criterion

Posted Date: March 11th, 2021

DOl: https://doi.org/10.21203/rs.3.rs-279205/v1

License: (1) This work is licensed under a Creative Commons Attribution 4.0 International License.

Read Full License 


\section{Title}

Arterial spin labeling: Adaptive thresholding for epileptogenic zone detection in MRI-negative epilepsy

Martin Gajdoš ${ }^{1}$, Pavel Říha ${ }^{1,2}$, Martin Kojan ${ }^{1}$, Irena Doležalová ${ }^{2}$, Henk-Jan Mutsaerts ${ }^{3,4}$, Jan Petr ${ }^{5}$, Ivan Rektor*1,2

${ }^{1}$ CEITEC - Central European Institute of Technology, Neuroscience Center, Masaryk University, Brno, Czech Republic

${ }^{2}$ Brno Epilepsy Center, Department of Neurology, St. Anne's University Hospital and Medical Faculty of Masaryk University, Brno, Czech Republic

${ }^{3}$ Department of Radiology and Nuclear Medicine, Amsterdam University Medical Center, VU University Medical Center, Amsterdam Neuroscience, Amsterdam, the Netherlands

${ }^{4}$ Department of Radiology and Nuclear Medicine, University Hospital Ghent, Ghent, Belgium

${ }^{5}$ Helmholtz-Zentrum Dresden-Rossendorf, Institute of Radiopharmaceutical Cancer Research, Dresden, Germany

* Corresponding Author:

Ivan Rektor

Brno Epilepsy Center

Department of Neurology

St. Anne's University Hospital

Pekařská 53 Brno 65691

Czech Republic

e-mail: irektor@med.muni.cz

Tel: +420603864046

\section{Acknowledgments:}

Support was provided by the AZV project 17-32292A from the Ministry of Health of the Czech Republic. We acknowledge the core facility MAFIL of CEITEC MU supported by the MEYS CR (LM2018129 CzechBiolmaging). We acknowledge Marek Bartoň and Michaela Bartoňová for help with preparation of a customized mask of intracranial volume. We thank Anne Johnson for the language correction. 


\section{Abstract}

Drug-resistant epilepsy is a diagnostic and therapeutic challenge, mainly in patients with negative MRI findings. State-of-the-art imaging methods complement standard epilepsy protocols with new information and help to epileptologists to increase reliability of their decisions. In this study, we investigate if arterial spin labeling (ASL) perfusion MRI can help localizing the epileptogenic zone (EZ). To that end, we developed an image processing method to detect the EZ as an area with hypoperfusion relative to the contralateral unaffected side, using subject-specific thresholding of the asymmetry index in ASL images. We demonstrated three thresholding criteria (minimal product criterion, minimal distance criterion, and elbow criterion) on 29 patients with MRI-negative epilepsy (age $32.98 \pm 10.4$ years). The minimal product criterion showed optimal results in terms of positive predictive value (mean 0.12 and 0.22 ) and true positive rate (mean 0.71 and 1.82). Additionally, we found high accuracy in determining the EZ side (mean 0.86 and 0.73 out of 1.00 ). In conclusion, the ASL can be easily incorporated to the standard presurgical MR protocol and it provides an additional benefit in EZ localization.

\section{Introduction}

Epilepsy is a neurological disease with a prevalence of about $1 \%$. About one third of the patients diagnosed with epilepsy do not respond to standard antiepileptic medication ${ }^{1}$. Epilepsy surgery is optimal in these drug-resistant patients. Unfortunately, no identifiable epileptogenic lesion is found in $20 \%$ to $40 \%$ of epilepsy surgery candidates using the anatomical magnetic resonance imaging (MRI) that is part of the standard presurgical MRI protocol ${ }^{2}$. Therefore, it is necessary to extend the structural imaging in these cases with other techniques measuring the physiology and pathophysiology of the brain.

The localization of the epileptogenic zone (EZ) is currently based on the convergence of clinical results, interictal/ictal EEG, and imaging techniques. Regional brain perfusion is one of the physiological parameters that may contribute to the EZ localization, particularly in MRI-negative epilepsy ${ }^{3}$.

Alterations in brain physiology can be measured by interictal and ictal single-photon emission tomography (SPECT) and their subtraction (subtraction ictal SPECT coregistered to MRI - SISCOM), and fluorodeoxyglucose positron emission tomography (FDG-PET). However, these methods have their limitations, including the use of radiotracers, higher costs, and the necessity of injecting the tracer at the very beginning of the seizure in SISCOM imaging. An alternative method for imaging perfusion that is approaching clinical adoption is arterial spin labeling (ASL) MRI. ASL is generally accessible as a product sequence on most MRI machines, is completely non-invasive, and has clinically feasible scanning times. Recent progress in the standardization of ASL acquisition ${ }^{4}$, data sharing, and processing ${ }^{5}$, made this a common tool for clinical research with increasing adoption into clinical practice ${ }^{6}$. ASL was demonstrated to be helpful in several types of epilepsy, including e.g. temporal lobe epilepsy ${ }^{7,8}$ and frontal lobe epilepsy ${ }^{8,9}$. One major remaining hurdle to the application of ASL as detection of EZ, is the definition of EZ on ASL perfusion images in a robust way; especially considering the physiological variability between patients ${ }^{10}$ and inter-scanner quantification challenges ${ }^{5}$. In this study, we focused on EZ detection in MRI-negative epilepsy based on ASL data. ASL has been proven to be useful in EZ localization in this type of epilepsy ${ }^{3,10}$.

The main problem associated with ASL is the strong inter-individual variability in perfusion data. In the past, some studies tried to overcome this issue using variability modeling ${ }^{10}$. Subject-specific thresholding might also be beneficial. We tried to solve this problem using subject data as control data. 
Our aim was to propose a straightforward and simple EZ localization workflow for ASL data that is feasible in a clinical environment and makes it possible to identify EZ with high robustness. In addition to accurate preprocessing, an important step of the analysis is the optimal thresholding of perfusion data ensuring both high sensitivity and specificity. To that end, we proposed a method that identifies EZ using an asymmetry index of cerebral blood flow (CBF) and compared three approaches to obtaining a subject-specific threshold based on an exponential fit to a normalized histogram of the CBF. Our proposed method is independent on selection of the parcellation scheme. Moreover, acquisition of healthy controls data for calibration is not needed. We tested the proposed approach for EZ detection in two groups of epilepsy surgery candidates with MRI-negative epilepsy. First, we tested the proposed method retrospectively in patients who underwent successful resection with satisfying results. Second, we prospectively tested the method in a group of presurgical candidates, in whom the hypothesis about EZ was based on a standard presurgical evaluation.

\section{Methods}

Our approach was divided into two steps. First, we optimized EZ localization in the group that had already had surgery (POST group). The ASL results in this group were confirmed by positive histopathological findings and successful surgery. Second, the optimized protocol was prospectively tested in the PRE group, in whom the EZ localization was established based on the clinical hypothesis after completing the standard evaluation protocol.

\section{Subjects}

We evaluated the data of 29 patients with drug-resistant epilepsy who were MRI-negative and underwent a complete presurgical evaluation at the Brno Epilepsy Center between 2017 and 2020. The MRI-negative epilepsy definition was based on a routine 3T MRI examination according to a presurgical epilepsy protocol evaluated by an experienced neuroradiologist ${ }^{11}$. We included all eligible patients with clinically normal MRI. The patients with visible anomalies were excluded from the study.

The study was approved by Ethics committee of the Masaryk University and by the Ethics committee of the St. Anne's University Hospital. This study was designed in accordance with the Declaration of Helsinki. All patients gave their informed consent before entering the study.

The standard protocol for presurgical evaluation included the following examination: (1) interictal and ictal EEG, (2) 3T MRI, (3) qualitative analysis of FDG-PET, (4) neuropsychological assessment, and (5) interictal/ictal SPECT and their post-processing SISCOM (SISCOM was performed only in clinically indicated patients).

The patients were divided into two groups: the preoperative group (PRE group) and the postoperative group (POST group).

In the PRE group, there were 22 epilepsy surgery candidates (mean $34 \pm 10.2$ years, 8 females). In the PRE group, the hypothesis on EZ localization was based on the standard protocol as defined above. We defined 10 possible areas of EZ: the right- or left-sided insula, frontal, temporal, parietal and occipital lobes. The masks were created according to the AAL atlas ${ }^{12}$. ROIs belonged to one of the ten defined areas.

In the POST group, there were 7 patients (mean $32 \pm 10.3$ years, 3 females) who underwent epilepsy surgery with complete or substantial seizure cessation characterized according to the International League Against Epilepsy (ILAE) ${ }^{13}$ as class 1 ( 5 patients) or class 2 ( 2 patients). In all patients, the invasive EEG was performed as a part of the presurgical evaluation. The EZ localization was confirmed by 
positive histopathology ${ }^{14,15}$. Based on the resection borders in post-resection MRI, we delineated the resection mask for each patient and approximated it for calculations as the mask of EZ.

\section{Data acquisition}

MRI data were acquired with a 3.0 T Magnetom Siemens Prisma. Perfusion imaging was performed using pseudo-continuous ASL (PCASL) with 2D EPI readout without background suppression ${ }^{16}$. We acquired 41 pairs of control and label images; each image consisted of 21 axial slices with $0.6 \mathrm{~mm}$ gap between slices, slice thickness $=6 \mathrm{~mm}$, repetition time $(T R)=4079 \mathrm{~ms}$, echo time $(T E)=16 \mathrm{~ms}, F O V=$ $192 \times 192 \mathrm{~mm}$, flip angle $(F A)=90^{\circ}$, matrix size $=64 \times 64$, bandwidth $=2056 \mathrm{~Hz} / \mathrm{Px}$, echo train length $=$ 48, post labeling delay (PLD) $=1800 \mathrm{~ms}$, labeling duration $(L D)=1800 \mathrm{~ms}$, and slice readout time $30 \mathrm{~ms}$. For quantification calibration, we acquired an $\mathrm{MO}$ scan with $\mathrm{TR}=8000 \mathrm{~ms}$. All perfusion data were acquired in 355s.

The MRI acquisition followed with a high-resolution anatomical T1-weighted image using the MPRAGE sequence with 240 sagittal slices, $T R=2300 \mathrm{~ms}, \mathrm{TE}=2.34 \mathrm{~ms}$, FOV $=256 \times 260 \mathrm{~mm}$, flip angle $=8^{\circ}$, matrix size $256 \times 260$, slice thickness $=1 \mathrm{~mm}$, bandwidth $=190 \mathrm{~Hz} / \mathrm{Px}$.

\section{Data preprocessing}

Preprocessing of ASL data was performed with the ExploreASL pipeline ${ }^{5}$ and consisted of motion correction, exclusion of motion outliers using the ENABLE algorithm ${ }^{17}$, CBF quantification according to the consensus paper ${ }^{4}$, and partial volume correction by linear regression ${ }^{18}$.

We calculated a voxelwise between-hemisphere asymmetry index (AI) ) of the CBF maps in voxel $x^{19}$ :

$$
\mathrm{Al}_{\mathrm{x}}=100 *\left(\mathrm{CBF}_{\mathrm{x}}-\mathrm{CBF}_{\mathrm{y}}\right) /\left(\mathrm{CBF}_{\mathrm{x}}+\mathrm{CBF}_{\mathrm{y}}\right)
$$

Here $y$ is position of the corresponding voxel in the contralateral hemisphere. We used only negative Al values, which represents interictal hypoperfusion. Finally, Al values were spatially smoothed with $8 \mathrm{~mm}$ FWHM. The Al map was calculated only within the a priori mask of intracranial volume.

\section{Proposed thresholding methods}

EZ zone is localized by thresholding of the AI maps. To reach an optimal performance of the method, with respect to its sensitivity and specificity, we proposed and compared three robust methods for subject-specific adaptive thresholding.

For all three methods, we computed a subject-specific Al histogram, normalized its count (nC) and Al range (nAl) axis in a range from 0 to 1 , and fitted the exponential curve $f$. All proposed thresholding methods for adaptive thresholding of Al are based on parameters of $f$; particularly, the threshold is based on:

(1) the minimal distance to point of origin $[0,0]$ (minimal distance criterion);

(2) the minimal product of normalized $\mathrm{nC}$ and $\mathrm{nAl}$ (minimal product criterion);

(3) the elbow criterion, i.e. the point at which $f$ bends. Briefly, $f$ bends at the point where the gradient of its tangent line is equal to -1 , which represents a -45 degree slope of the line.

Example of thresholds and resulting clusters based on proposed criteria is shown in Figure 1. 
A

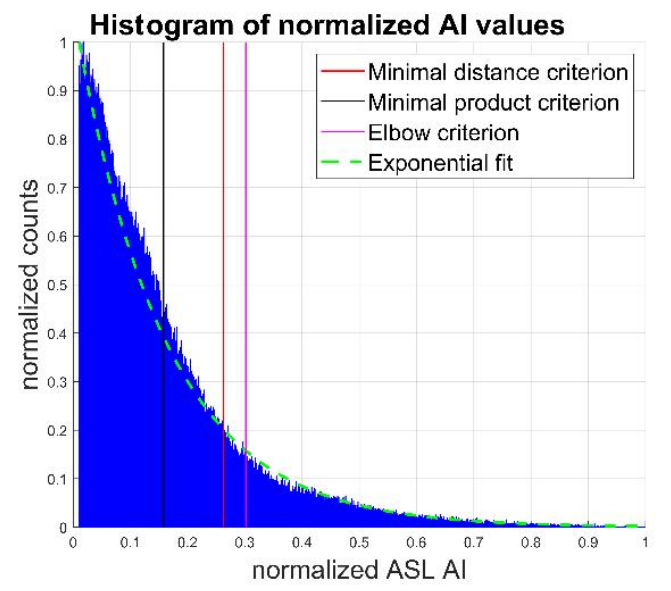

B

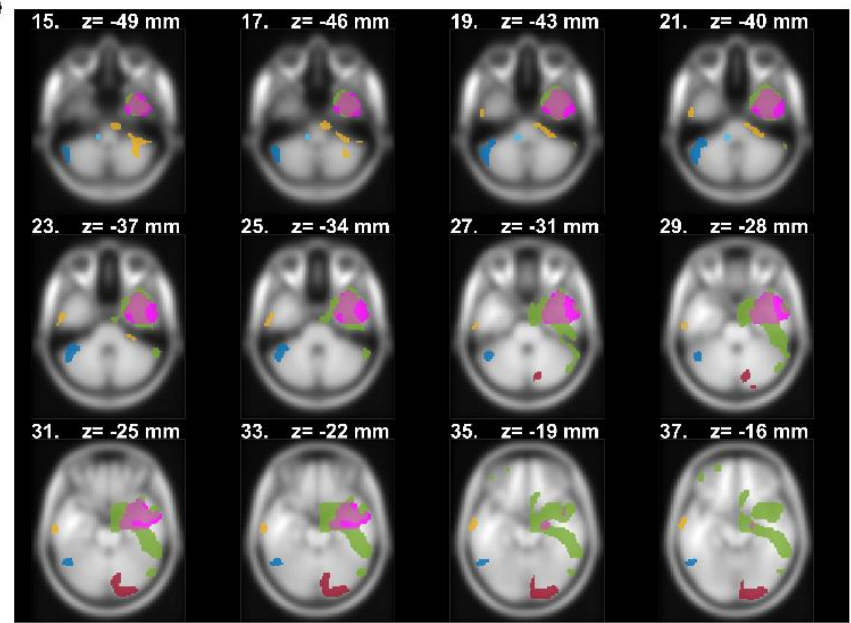

Figure 1. Example of $\mathrm{Al}(\mathrm{A})$ histogram of a representative subject from the POST group. Adaptive thresholds are depicted, based on the three proposed thresholding methods. On the right (B) is a slice view with a resection mask (magenta) and clusters (other colors) of Al after thresholding with the minimal product criterion. This was revealed to be the optimal criterion. Used abbreviations: Al asymmetry index, ASL - arterial spin labeling.

\section{Metrics for evaluation of accuracy}

After thresholding the Al images, we only kept the connected clusters with more than 500 voxels ( $1.7 \mathrm{ml})$ for further analyses. To evaluate the accuracy we used the following metrics:

(A) Mask hit; a binary criterion that is true only if at least one cluster covers more than $10 \%$ of the resection mask (or more than $1 \%$ of the EZ hypothesis mask);

(B) Percent of overlay of the resection mask or the mask of EZ hypothesis by the most precise cluster;

(C) Precision in determining the EZ side. The predicted EZ side is decided according to greater sum of suprathresholded Al voxels in the left and right hemispheres. The criterion is binary;

(D) True positive (TP). Number of clusters covering more than $10 \%$ of the resection mask or more than $1 \%$ of the EZ hypothesis mask;

(E) False positive (FP). Number of clusters outside of the resection/EZ hypothesis mask or covering less than $10 \%$ of the resection mask or less than $1 \%$ of the EZ hypothesis mask;

(F) Positive predictive value (PPV). Computed as TP divided by sum of TP and FP.

\section{Results}

When comparing the thresholding methods, the minimal product criterion corresponded on average to $17 \%$ of the highest Al values. The minimal distance criterion and the elbow criterion are stricter. The minimal distance criterion corresponded on average to $6 \%$, and the elbow criterion corresponded to $4 \%$ of the highest Al values.

The best mask hit was achieved with the minimal product criterion threshold with $71 \%$ true positive values for the POST group and $100 \%$ true values for the PRE group. The minimal distance criterion and the elbow criterion reached lower amounts of the true values. In both groups, TP was the highest for minimal product criterion, but the lowest FP was achieved with the elbow criterion. The optimal tradeoff was expressed by PPV, which was in all cases optimal for the minimal product criterion. Details on the performances of all of the used metrics are depicted in Table 1. 


\begin{tabular}{|c|c|c|c|c|c|c|}
\hline \multirow[b]{2}{*}{$\begin{array}{l}\text { Metric / } \\
\text { thresholding } \\
\text { method }\end{array}$} & \multicolumn{3}{|c|}{ PRE group } & \multicolumn{3}{|c|}{ POST group } \\
\hline & $\begin{array}{l}\text { Minimal } \\
\text { distance } \\
\text { criterion }\end{array}$ & $\begin{array}{l}\text { Minimal } \\
\text { product } \\
\text { criterion }\end{array}$ & $\begin{array}{l}\text { Elbow } \\
\text { criterion }\end{array}$ & $\begin{array}{l}\text { Minimal } \\
\text { distance } \\
\text { criterion }\end{array}$ & $\begin{array}{l}\text { Minimal } \\
\text { product } \\
\text { criterion }\end{array}$ & $\begin{array}{l}\text { Elbow } \\
\text { criterion }\end{array}$ \\
\hline Mask hit & $\begin{array}{l}0.73 \pm 0.46 \\
(1.00)\end{array}$ & $\begin{array}{l}1.00 \pm 0.00 \\
(1.00)\end{array}$ & $\begin{array}{l}0.68 \pm 0.48 \\
(1.00) \\
\end{array}$ & $\begin{array}{l}0.57 \pm 0.54 \\
(1.00) \\
\end{array}$ & $\begin{array}{l}0.71 \pm 0.49 \\
(1.00)\end{array}$ & $\begin{array}{l}0.43 \pm 0.54 \\
(0.00)\end{array}$ \\
\hline $\begin{array}{l}\text { Percent of overlay } \\
\text { of the resection } \\
\text { mask/mask of EZ } \\
\text { hypothesis by the } \\
\text { most precise } \\
\text { cluster }\end{array}$ & $\begin{array}{l}5.00 \pm 4.49 \\
(3.96)\end{array}$ & \begin{tabular}{|l}
$15.14 \pm 9.74$ \\
$(13.07)$
\end{tabular} & $\begin{array}{l}3.35 \pm 3.38 \\
(1.96)\end{array}$ & $\begin{array}{l}17.82 \pm \\
20.03 \\
(12.19)\end{array}$ & $\begin{array}{l}31.98 \pm 22.83 \\
(35.74)\end{array}$ & $\begin{array}{l}14.38 \pm \\
18.31 \\
(9.72)\end{array}$ \\
\hline $\begin{array}{l}\text { Precision in } \\
\text { determining the } \\
\text { EZ side }\end{array}$ & $\begin{array}{l}0.68 \pm 0.48 \\
(1.00)\end{array}$ & $\begin{array}{l}0.73 \pm 0.46 \\
(1.00)\end{array}$ & $\begin{array}{l}0.68 \pm 0.48 \\
(1.00)\end{array}$ & $\begin{array}{l}0.86 \pm \\
0.38(1.00)\end{array}$ & $\begin{array}{l}0.86 \pm 0.38 \\
(1.00)\end{array}$ & $\begin{array}{l}0.86 \pm \\
0.38(1.00)\end{array}$ \\
\hline TP & $\begin{array}{l}1.32 \pm 1.04 \\
(1.00)\end{array}$ & $\begin{array}{l}1.82 \pm 0.96 \\
(2.00)\end{array}$ & $\begin{array}{l}1.00 \pm 0.87 \\
(1.00) \\
\end{array}$ & $\begin{array}{l}0.57 \pm 0.54 \\
(1.00) \\
\end{array}$ & $\begin{array}{l}0.71 \pm 0.49 \\
(1.00)\end{array}$ & $\begin{array}{l}0.43 \pm 0.54 \\
(0.00)\end{array}$ \\
\hline FP & $\begin{array}{l}5.73 \pm 1.64 \\
(6.00)\end{array}$ & $\begin{array}{l}6.64 \pm 2.15 \\
(6.00)\end{array}$ & $\begin{array}{l}4.91 \pm \\
1.74(5.00)\end{array}$ & $\begin{array}{l}6.57 \pm 1.62 \\
(7.00)\end{array}$ & $\begin{array}{l}6.29 \pm 1.89 \\
(6.00)\end{array}$ & $\begin{array}{l}4.86 \pm \\
2.04(4.00)\end{array}$ \\
\hline PPV & $\begin{array}{l}0.18 \pm 0.14 \\
(0.17)\end{array}$ & $\begin{array}{l}0.22 \pm 0.11 \\
(0.19)\end{array}$ & $\begin{array}{l}0.16 \pm 0.14 \\
(0.17)\end{array}$ & $\begin{array}{l}0.07 \pm 0.07 \\
(0.11)\end{array}$ & $\begin{array}{l}0.12 \pm 0.08 \\
(0.14)\end{array}$ & $\begin{array}{l}0.07 \pm 0.09 \\
(0.00)\end{array}$ \\
\hline
\end{tabular}

Table 1. Evaluation of particular metrics in proposed thresholding methods. The values are mean \pm standard deviation (median). The optimal values are marked in bold. Used abbreviations: TP - true positive, FP - false positive, PPV - positive predictive value, EZ - epileptogenic zone, POST postoperative group, PRE - preoperative group.

\section{Discussion}

Our data confirm the applicability of ASL in MRI-negative epilepsy. We proposed a data-driven approach for EZ localization that is easily applicable in clinical praxis. The main advantages of this approach are that (1) it is independent of a priori hypotheses about the parcellation scheme, such as the ROI-based analysis using the Harvard-Oxford atlas ${ }^{10}$, and (2) it does not require data from healthy controls as a calibration group for the perfusion template.

The main objective of our paper was to define the optimal way to use CBF for detection of EZ in MRInegative epilepsy. This process, as done by Al computation, leads to the suppression of inter-subject variability in more advanced steps than the variability modeling approach. Additionally, the datadriven approach of adaptative thresholding suppresses part of inter-individual variability.

Interestingly, we observed high accuracy when determining the lateralization (right-sided vs. leftsided) of EZ. In the POST group, all three criteria identified the EZ side with identically high levels of accuracy. This accuracy reached $88 \%$ and failed in only one case. In the PRE group, the accuracy decreased to $69 \%$; this level was reached by both the minimal product criterion and the elbow criterion. Although all of the criteria had similar accuracy in EZ lateralization (i.e., in determining whether EZ was in the left or right hemisphere), the choice of an optimal criterion is crucial for EZ localization. 
The results of the metrics for evaluating the precision of the thresholding methods were in concordance in both groups. The mask hit metric represents the sensitivity of Al values to designate the EZ resection mask or EZ hypothesis mask. This metric was high for all of the tested thresholding methods, but it reached the best values for the minimal product criterion. Because Al on perfusion maps is not intended as single decisive method for EZ localization and is characterized with high sensitivity, it is crucial to combine Al-based EZ detection with other methods from the standard presurgical clinical protocol. This can reasonably help in refining the final decision of the EZ location in epilepsy surgery candidates.

TP, the analogical metric to mask hit, is also optimal for the minimal product criterion. Interestingly, identical results for the POST group were caused with maximally one cluster emerging to cover at least $10 \%$ of the resection mask. In the PRE group, more clusters could meet the criterion and thus the information was not binary. FP is optimal for the elbow criterion, but when considering PPV, the optimal tradeoff between TP and FP, the minimal product criterion was optimal.

Although the minimal product criterion is more benevolent, the number of misplaced clusters was only slightly increased in comparison to other proposed thresholding methods. Moreover, when considering the higher ability of resection mask detection (mask hit), we suggest using the minimal product criterion as the optimal thresholding method of the presented approaches.

A limitation of our approach is a weakness of the Al: the inability to detect symmetrical disruptions in perfusion (e.g. bitemporal epilepsy). For this special case would be more suitable testing CBF data to the perfusion template based on healthy controls data. Second limitation is number of subjects in the POST group: only in part of the epilepsy surgery candidates reached the epileptologists sufficiently reliable decision on EZ localization.

To conclude, based on mask hit and PPV, the minimal product criterion is optimal for adaptive thresholding of Al. In this study, we presented novel method for EZ localization based on perfusion data obtained with ASL sequences. We believe this method is suitable for use in clinical environments. In our opinion, Al indexes computed from ASL data appear to be helpful in EZ localization, especially in combination with other methods from the standard clinical presurgical protocol. This could contribute to EZ localization and even difficult cases of MRI-negative epilepsy could be tractable.

\section{Bibliography}

1. Stafstrom, C. E. \& Carmant, L. Seizures and Epilepsy: An Overview for Neuroscientists. Cold Spring Harb. Perspect. Med. 5, a022426-a022426 (2015).

2. Leeman-Markowski, B. Review of MRI-Negative Epilepsy. JAMA Neurol. 73, 1377 (2016).

3. Boscolo Galazzo, I. et al. Cerebral metabolism and perfusion in MR-negative individuals with refractory focal epilepsy assessed by simultaneous acquisition of 18 F-FDG PET and arterial spin labeling. Neurolmage Clin. 11, 648-657 (2016).

4. Alsop, D. C. et al. Recommended implementation of arterial spin-labeled perfusion MRI for clinical applications: A consensus of the ISMRM perfusion study group and the European consortium for ASL in dementia. Magn. Reson. Med. 73, 102-116 (2015).

5. Mutsaerts, H. J. M. M. et al. ExploreASL: An image processing pipeline for multi-center ASL perfusion MRI studies. Neuroimage 219, 117031 (2020).

6. Manfrini, E. et al. From research to clinical practice: a European neuroradiological survey on quantitative advanced MRI implementation. Eur. Radiol. (2021). doi:10.1007/s00330-02007582-2 
7. Guo, X. et al. Asymmetry of cerebral blood flow measured with three-dimensional pseudocontinuous arterial spin-labeling mr imaging in temporal lobe epilepsy with and without mesial temporal sclerosis. J. Magn. Reson. Imaging 42, 1386-1397 (2015).

8. Storti, S. F. et al. Combining ESI, ASL and PET for quantitative assessment of drug-resistant focal epilepsy. Neuroimage 102, 49-59 (2014).

9. Sierra-Marcos, A. et al. Accuracy of arterial spin labeling magnetic resonance imaging (MRI) perfusion in detecting the epileptogenic zone in patients with drug-resistant neocortical epilepsy: comparison with electrophysiological data, structural MRI, SISCOM and FDG-PET. Eur. J. Neurol. 23, 160-167 (2016).

10. Boscolo Galazzo, I. et al. Patient-Specific Detection of Cerebral Blood Flow Alterations as Assessed by Arterial Spin Labeling in Drug-Resistant Epileptic Patients. PLoS One 10, e0123975 (2015).

11. Commission on Diagnostic Strategies Recommendations for Functional Neuroimaging of Persons with Epilepsy. Epilepsia 41, 1350-1356 (2000).

12. Tzourio-Mazoyer, N. et al. Automated anatomical labeling of activations in SPM using a macroscopic anatomical parcellation of the MNI MRI single-subject brain. Neuroimage 15, $273-$ 89 (2002).

13. Wieser, H. G. et al. ILAE Commission Report. Proposal for a new classification of outcome with respect to epileptic seizures following epilepsy surgery. Epilepsia 42, 282-6 (2001).

14. Blümcke, I. et al. The clinicopathologic spectrum of focal cortical dysplasias: A consensus classification proposed by an ad hoc Task Force of the ILAE Diagnostic Methods Commission1. Epilepsia 52, 158-174 (2011).

15. Blümcke, I. et al. International consensus classification of hippocampal sclerosis in temporal lobe epilepsy: A Task Force report from the ILAE Commission on Diagnostic Methods. Epilepsia 54, 1315-1329 (2013).

16. Li, X. et al. Theoretical and experimental evaluation of multi-band EPI for high-resolution whole brain pCASL Imaging. Neuroimage 106, 170-181 (2015).

17. Shirzadi, Z. et al. Enhancement of automated blood flow estimates (ENABLE) from arterial spinlabeled MRI. J. Magn. Reson. Imaging 47, 647-655 (2018).

18. Asllani, I., Borogovac, A. \& Brown, T. R. Regression algorithm correcting for partial volume effects in arterial spin labeling MRI. Magn. Reson. Med. 60, 1362-1371 (2008).

19. Lim, Y.-M. et al. Usefulness of pulsed arterial spin labeling MR imaging in mesial temporal lobe epilepsy. Epilepsy Res. 82, 183-189 (2008).

\section{Author contributions statement}

All authors reviewed the manuscript.

Martin Gajdoš: Wrote the main manuscript text, prepared conception of the work, analyzed the ASL data, developed novel methodology, and interpreted the data.

Pavel Říha: Participated on acquisition of the data, analyses of the data and development of novel methodology 
Martin Kojan: Participated on interpretation of the data, analysed the data

Irena Doležalová: Interpreted the data, substantially revised the work

Henk-Jan Mutsaerts: Prepared new software used in the work, substantially revised the work

Jan Petr: Prepared new software used in the work, substantially revised the work

Ivan Rektor: Interpreted the data, substantially revised the work

\section{Legends}

Figure 1. Example of Al (A) histogram of a representative subject from the POST group. Adaptive thresholds are depicted, based on the three proposed thresholding methods. On the right (B) is a slice view with a resection mask (magenta) and clusters (other colors) of Al after thresholding with the minimal product criterion. This was revealed to be the optimal criterion. Used abbreviations: Al asymmetry index, ASL - arterial spin labeling.

Table 1: Evaluation of particular metrics in proposed thresholding methods. The values are mean \pm standard deviation (median). The optimal values are marked in bold. Used abbreviations: TP - true positive, FP - false positive, PPV - positive predictive value, EZ - epileptogenic zone, POST postoperative group, PRE - preoperative group.

\section{Additional Information}

\section{Competing interests statement}

The authors declare no competing interests. 


\section{Figures}

A

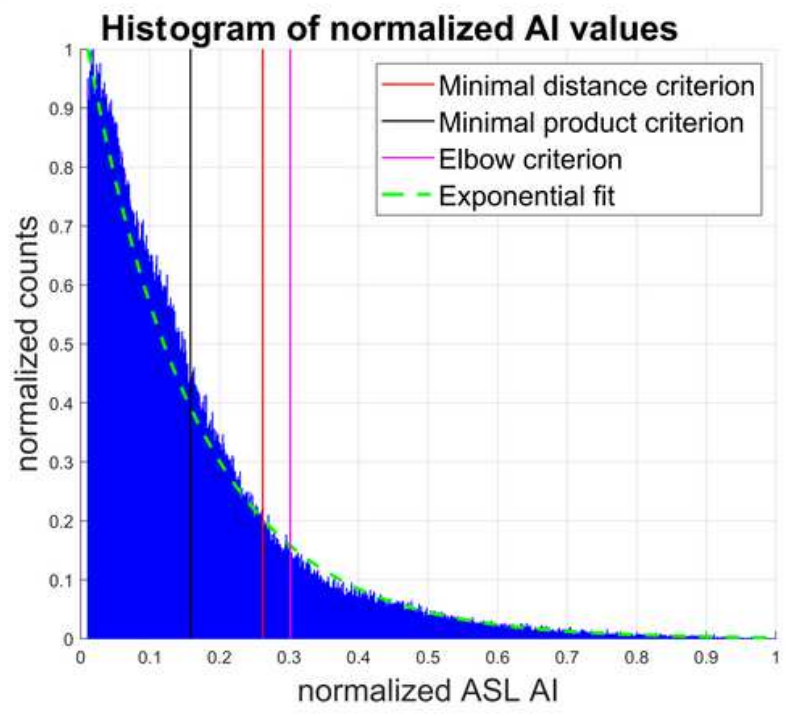

B

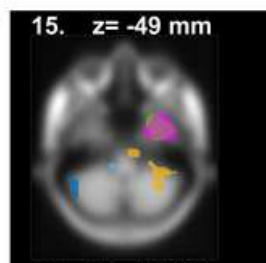

23. $z=-37 \mathrm{~mm}$

25. $z=-34 \mathrm{~mm}$

27. $z=-31 \mathrm{~mm}$

29. $z=-28 \mathrm{~mm}$

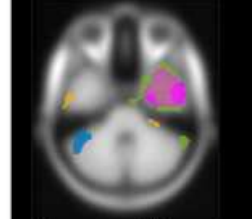

31. $\mathrm{z}=-25 \mathrm{~mm}$

33. $\mathrm{z}=-22 \mathrm{~mm}$

35. $\mathrm{z}=-19 \mathrm{~mm}$

37. $\mathrm{z}=-16 \mathrm{~mm}$

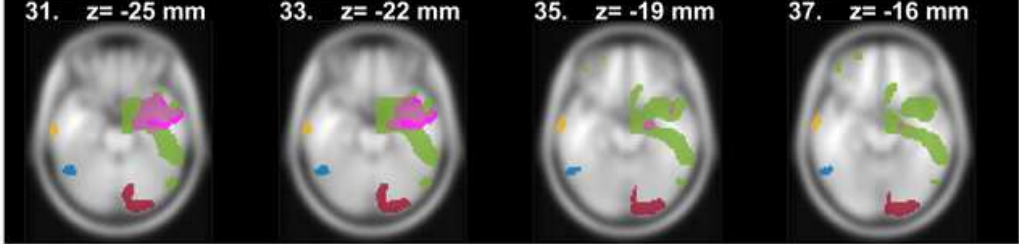

\section{Figure 1}

Example of $\mathrm{Al}(\mathrm{A})$ histogram of a representative subject from the POST group. Adaptive thresholds are depicted, based on the three proposed thresholding methods. On the right (B) is a slice view with a resection mask (magenta) and clusters (other colors) of Al after thresholding with the minimal product criterion. This was revealed to be the optimal criterion. Used abbreviations: Al - asymmetry index, ASL arterial spin labeling. 